\title{
Demographic characteristics of children with early clinical manifestation of inflammatory bowel disease
}

\author{
Edyta Szymańska1, Sylwia Szymańska², Michał Szczepański ${ }^{3}$, Piotr Landowski ${ }^{4}$, Grażyna Czaja-Bulsa ${ }^{5}$, \\ Elżbieta Jarocka-Cyrta ${ }^{6}$, Bartosz Korczowski ${ }^{7}$ Elżbieta Krzesiek ${ }^{8}$, Jarosław Kierkuś ${ }^{3}$

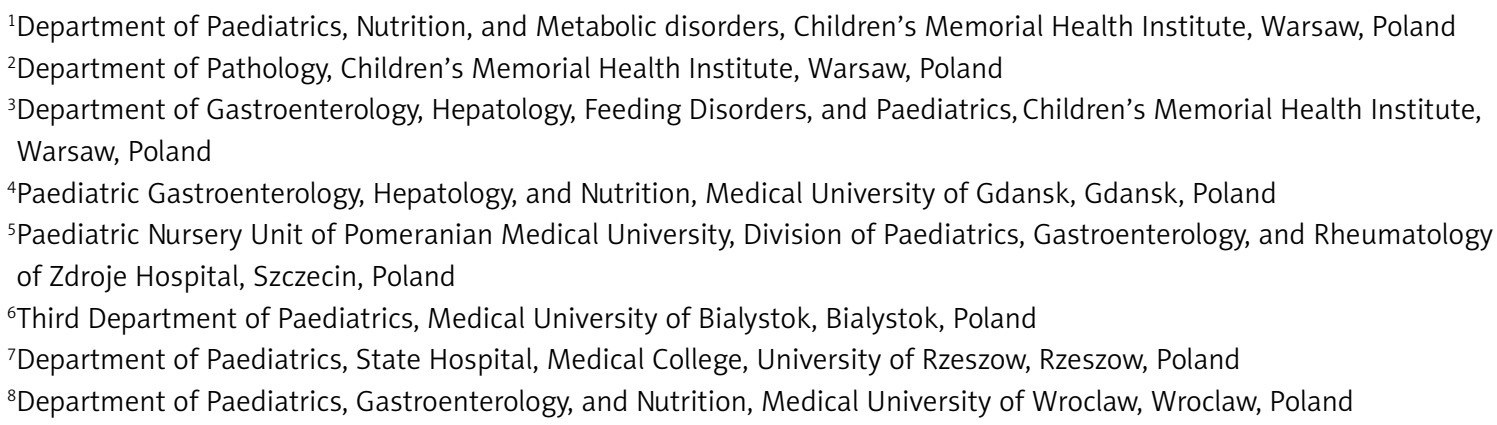

Key words: inflammatory bowel disease, early onset, children.

Address for correspondence: Jarosław Kierkuś MD, PhD, Department of Gastroenterology, Hepatology, Feeding Disorders, and Paediatrics, Children's Memorial Heath Institute, Al. Dzieci Polskich 20, 04-730 Warsaw, Poland, phone/fax: +48 2281573 92, e-mail: j.kierkus@czd.pl

\begin{abstract}
Introduction: Inflammatory bowel disease (IBD), which includes Crohn's disease (CD) and ulcerative colitis (UC), is a chronic condition of the colon and small intestine. The disease is common in young people (children and young adults), but it is rare in children younger than five years of age. Therefore, IBD developing during the first years of life (under the age of 5) is known as an early-onset IBD (EO-IBD), and it is considered to be a specific entity with a distinct phenotype. However, the available data on that issue are still insufficient.

Aim: To determine the characteristics and clinical course of children with early-onset IBD.

Material and methods: We performed a retrospective database analysis of 47 infants younger than 5 years old diagnosed with IBD. Patient's demographic data, including age, sex, and age at disease onset, were collected in 6 paediatric hospitals in Poland. Disease location was established on the basis of the review of all endoscopic, colonoscopic, histopathological, and radiological records. All possible complications were reported, as well as any treatment and its efficacy. Since the diagnosis was established all patients have been on follow up.

Results: Among 47 children registered in the database, 23 (49\%) had a diagnosis of CD, 16 (34\%) had UC, and 8 (17\%) had IC (indeterminate colitis). The mean age at diagnosis was $28.5 \pm 27.5$ months; $57.4 \%$ were male. The most common location/type of disease was ileocolonic disease (L3). The most common complication of IBD was anaemia, found in 30 (63.8\%) children. The observed course of the disease was either severe or moderate. In 4 children younger than 2 years old, surgery was performed.

Conclusions: Inflammatory bowel disease in children younger than 5 years old includes UC, CD, and a relatively high proportion of IC. In early-onset IBD severe and moderate course of the disease is usually observed. Disease manifestation in these patients is predominantly ileocolonic.
\end{abstract}

\section{Introductions}

The onset of inflammatory bowel disease (IBD) peaks within the second decade of life, with approximately $20-35 \%$ of patients diagnosed in the paediatric age group [1, 2]. Inflammatory bowel disease includes ulcerative colitis (UC) and Crohn's disease (CD). According to recent reports $[3,4]$ the incidence of paediatric CD has increased, although the incidence of UC has remained stable [5]. Not only is the number of patients increasing but there is also data indicating onset of IBD within the first years of life $[6,7]$. Inflammatory bow- 
el disease of very early onset includes, apart from UC and $C D$, a relatively high proportion of indeterminate colitis (IC). Paediatric onset IBD has unique phenotypic heterogeneity, responses to therapy, and prognosis. The manifestation of early-onset IBD has a very severe course and guarded prognosis with life-threatening symptoms [8, 9]. Overall the disease manifestations are primarily colonic, with severe perianal disease and severe extra-gastrointestinal manifestations, and it can rapidly progress to pancolitis [6]. Early-onset IBD is unique in its association to metabolic diseases, neutrophil defects, and immunodeficiency. Therefore, it is controversial whether this form belongs to $U C$ and $C D$ or is a presentation of the primary disease [10]. The aetiology of IBD is unknown. Recent data suggest that altered immune homeostasis within the intestinal mucosa, together with phagocytic function, metabolic pathways, environmental factors, and genetic predisposition play a role in its pathology. The last factor seems to play a fundamental role in the natural history of early-onset IBD. Current data suggest mutations in genes encoding interleukin-10 receptor, IL-27, MTMR3 (essential component of autophagy), and CAPN10 (necessary for the regulation of endoplasmic reticulum stress). In addition, the roles of PSMG1, TNFRSF6B, ZMIZ1, and SMAD3 are also discussed in relation to abnormal protein degradation and secondary immune response [11].

Inflammatory bowel disease in children younger than five years has a distinct phenotype and should be considered as a specific entity. There are only a few reports analysing the outcome of this paediatric subgroup. Longitudinal studies are needed to determine the clinical implications of early-onset IBD.

\section{Aim}

The aim of this study was to determine the characteristics and clinical course of children with early-onset IBD.

\section{Material and methods}

We performed a retrospective database analysis of 47 infants less than 5 years old, diagnosed with IBD at 6 paediatric centres in Poland: the Children's Memorial Health Institute, Warsaw; and hospitals in Bialystok, Szczecin, Rzeszow, Gdansk, and Wroclaw. Patient demographics, including age, sex, and age at disease onset, were tallied. Disease location was identified on the basis of a review of all endoscopic, colonoscopic, histopathological, and radiologic records. The Montreal Classification was used to classify the location of a disease.

All possible complications were reported. Patients were administered different medications such as prednisolone, azathioprine, 6-mercaptopurine, methotrexate, and antibiotics. Biological agents and surgery were also included. The efficacy of the applied treatment was assessed. Patients were followed up clinically to determine the extent of disease progression on the basis of the initial diagnosis of IBD.

\section{Results}

Among 47 children registered in the database $23(49 \%)$ had a diagnosis of CD, 16 (34\%) had UC, and $8(17 \%)$ had IC. The mean age at diagnosis was 28.5 \pm 27.5 months; $57.4 \%$ were male. One $(2.12 \%)$ patient had limited caecal disease (L1), 20 (42.5\%) had colon involvement (L2), and 25 (53.2\%) had ileocolonic location (L3), whether CD, UC, or IC. Upper gastrointestinal tract involvement was found in seven CD patients, but it was not isolated upper disease (L4) as they had simultaneously either colonic or ileocolonic lesions. In 1 (2.15\%) UC patient extra-intestinal manifestation of the disease was a presenting symptom. In this case the diagnosis of IBD was established on the base of a histopathological examination of the biopsy taken during endoscopy performed as part of a differential diagnosis. Table I presents demographic characteristic of 47 patients with EO-IBD, and Figure 1 presents the exact location of the disease in CD, UC, and IC patients individually. Anaemia, the most common complication, was detected in 30 (63.8\%) objects. Three (6.4\%) patients younger than 3 years of age developed strictures; 2 (4.2\%) patients younger than 1 year of age developed abscess; 1 (4.2\%) patient at the age of 4 months developed fistula. Most children were treated with mesalamine (93.6\%) and/or steroids (59.6\%) either orally or intravenously. Five CD patients younger than 3 years of age were administered infliximab and 4 of them had to undergo surgery. Figure 2 presents the types and incidences of administered treatments. Overall, response to the treatment was positive in most cases; the average number of flare-ups was one, except from 4 of the CD patients who were treated with therapy including biological agents and operation.

\section{Discussion}

The incidence and prevalence of IBD has risen during the last few decades even in young children. Although rare, IBD can begin in infancy [9]. Clinical data on very early-onset IBD are sparse as this group represents a small subset of paediatric IBD patients. The aim of our study was to determine the characteristics and clinical course of IBD in children younger than 5 years old. Some studies suggest that early-onset IBD has a distinct phenotype and should be considered as a specific entity [6, 10]. The prevalence of IC in this paediatric subgroup is higher than that observed in older children and adults. In our database the diagnosis of CD prevailed; however, there were eight (17\%) infants with IC, all of them 
Table I. Demographic characteristics of 47 children with early clinical manifestation of inflammatory bowel disease

\begin{tabular}{lccc} 
Parameter & CD patients & UC patients & IC patients \\
\hline Number of patients $(n)$ & $23 / 47$ & $16 / 47$ & $8 / 16$ \\
\hline Male & $11 / 23$ & $10 / 16$ & $5 / 8$ \\
\hline Mean age at diagnosis [months] & 19.7 (from 4 up to 56) & 19.6 (from 1 up to 56) & 11.7 (from 1 up to 32) \\
\hline Mean age at diagnosis [months] & & 19.6 (from 1 up to 56) & \\
\hline Complications: & & & $2 / 8$ \\
\hline None & $6 / 23$ & $9 / 16$ & $6 / 8$ \\
\hline Anaemia & $17 / 23$ & $7 / 16$ & $0 / 8$ \\
\hline Strictures & $3 / 23$ & $0 / 16$ & $0 / 8$ \\
\hline Fistula & $1 / 23$ & $0 / 16$ & $0 / 8$
\end{tabular}

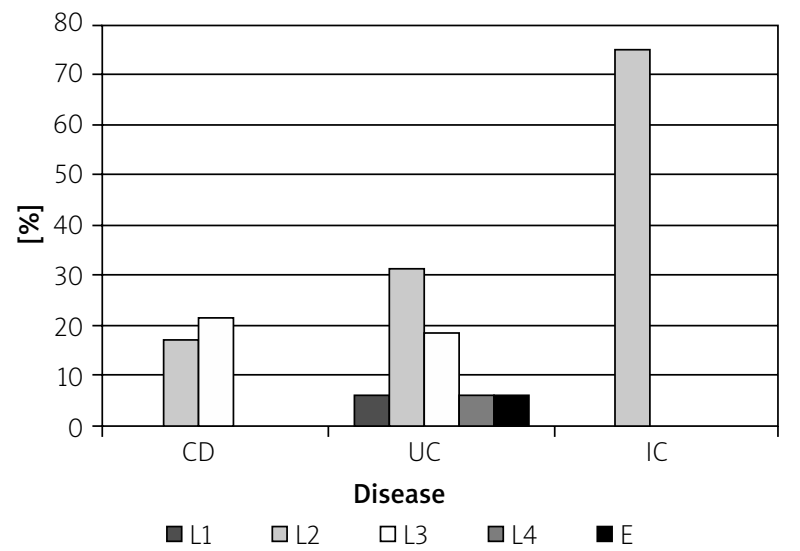

Figure 1. Location of the disease according to Montreal Classification

younger than 3 years of age. Diagnosis of IBD in this age group is particularly difficult. The differential diagnoses are infectious and allergic colitis, which are the major causes of infantile colitis. Rare conditions such as immune defects (e.g. septic granulomatosis) or GSD type $1 \mathrm{~b}$ can mimic IBD [12]. None of these were ruled out in our patients.

Some studies also suggested that IBD in infants follows a more severe course than in older patients, whereas other studies did not imply a similar poor prognosis $[6,9,13,14]$. Overall, the disease manifestations are primarily colonic and rapidly progress to pancolitis. In our study almost all of the 47 children had colitis, except 1 patient who presented only extra-intestinal manifestation. This colonic location of the disease in our patients was consistent with reports of Heymen et al. [9] and

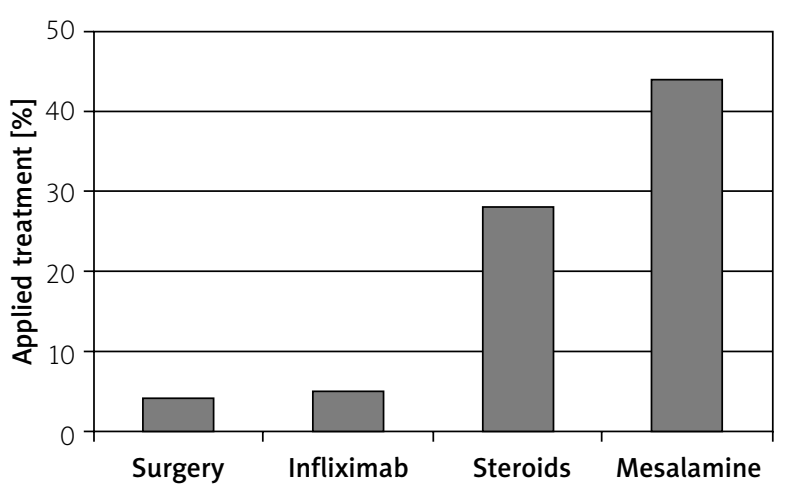

Figure 2. Types and incidence of administered treatments

Most often applied medication - mesalamine (44 patients). Steroids - 28 children (with/or without mesalamine). Infliximab -5 patients. Surgery -4 children (all of them had previously received infliximab).

Mamula et al. [15]. All of their patients also had colitis. We classified a disease location according to Paris Classification, which is a paediatric modification of Montreal Classification [16]. Only 17 (36.2\%) patients had isolated colonic disease (L2), while 8 (17\%) had ileocolonic disease (L3), and 20 (42.5\%) had pancolitis. It may be caused by the fact that very early-onset IBD rapidly progresses to pancolitis. Some studies report that IBD in infants has a very severe course with extra-gastrointestinal symptoms and guarded prognosis with life-threatening signs and needs an aggressive therapeutic approach [10]. In our study only one UC patient younger than 3 years of age had extra-gastrointestinal symptoms. In this case the diagnosis of IBD was established due to an endoscopy performed during the diagnostic process of presented symptoms. In 17 (36.2\%) children no compli- 
cations were observed, and the most common complication was anaemia, which was detected in $30(63.8 \%)$ patients. Three (6.4\%) patients younger than 3 years of age developed strictures, 2 (4.2\%) younger than one year of age developed abscess, and 1 (4.2\%) infant at the age of 4 months developed fistula. These were patients with severe and refractory conditions, who needed an aggressive therapeutic approach including a biological agent (infliximab). Four of them had to undergo surgery. Dokucu et al. [17] observed that the need for surgery was markedly higher and earlier in this specific paediatric subgroup. Overall, among 47 children, the course of the disease was severe or moderate. Almost all of our patients received mesalamine (93.6\%) and/or multiple immunosuppressive drugs (most often steroids either orally or intravenously). Thirty-eight (81\%) patients had a history of using antibiotics. Altered interaction between the normal gut flora and the intestinal mucosa is a major triggering factor for $\operatorname{IBD}[2,7,18]$, and some studies indicate a link between antibiotic use and the development of IBD $[19,20]$. Moreover, IBD in infancy can be associated with metabolic diseases, neutrophil defects, and immunodeficiency states [10], but it is still not known whether those patients have an immunological disorder or genetic basis of the disease.

Early-onset IBD has a distinct phenotype and presents a unique opportunity to study the impact of immunological status, gut microflora, metabolic pathways, and environmental factors on the genetic predisposition and the natural history of IBD.

\section{Conclusions}

Inflammatory bowel disease in children younger than 5 years includes UC and CD and a relatively high proportion of IC. In early-onset IBD severe and moderate course of the disease is apparent. Pathological changes are present mainly in the large intestine and only in single cases in the upper part of the alimentary tract and jejunum, and the disease rapidly progresses to pancolitis. Longitudinal studies are needed to determine the clinical implications of this paediatric IBD subgroup.

\section{Conflict of interest}

The authors declare no conflict of interest.

\section{References}

1. Sawczenko A, Sandhu BK, Logan RF, et al. Prospective survey of childhood inflammatory bowel disease in the British Isles. Lancet 2001; 357: 1093-4.

2. Auvin S, Molinié F, Gower-Rousseau C, et al. Incidence, clinical presentation and location at diagnosis of pediatric inflammatory bowel diseases: prospective population based study in northern France (1988-1999). J Pediatr Gastroenterol Nutr 2005; 4: 49-55.
3. Cosgrove M, AIAtia RF, Jenkins HR. The epidemiology of pediatric inflammatory bowel diseases. Arch Dis Child 1996; 74: 460-1.

4. Griffiths AM, Hugot JP. Inflammatory bowel disease. In: Pediatric gastrointestinal disease. 4th ed. Walker WA, Goulet O, Kleinman RE (eds.). BC Decker study Gut 2005; 357-63.

5. Gryboski JD. Crohn's disease in children 10 years old and younger: comparison with ulcerative colitis. J Pediatr Gastroenterol Nutr 1994; 18: 174-82.

6. Ruemmele FM, El Khoury MG, Talbotec C, et al. Characteristics of inflammatory bowel disease with onset during the first year of life. J Pediatr Gastroenterol Nutr 2006; 43: 603-9.

7. Dady IM, Thomas AG, Miller V, Kelsey AJ. Inflammatory bowel diseses in infancy: an increasing problem. J Pediatr Gastroenterol Nutr 1996; 23: 569-74.

8. Marx G, Seidman E, Martin S, Deslandres C. Outcome of Crohn's diseases diagnosed before two years of age. J Pediatr 2002; 140: 470-3.

9. Heymen MB, Kirshner BS, Gold BD, et al. Children with early onset inflammatory bowel disease: analysis of a pediatric IBD consortium registry. J Pediatr 2005; 146: 35-40.

10. Lebenthal E, Branski D, Walker-Smith JA, et al. Pediatric and inflammatory bowel disease: perspective and consequences. Pediatr Adolesc Med 2009; 14: 67-75.

11. Henderson P, van Limbergen JE, Wilson DC, et al. Genetics of childhood-onset inflammatory bowel disease. Inflamm Bowel Dis 2011; 17: 346-61.

12. Zimmer KP. Inflammatory bowel disease in infants: the other end of the beginning. J Pediatr Gastroenterol Nutr 2006; 43: 566-8.

13. Cannioto Z, Berti I, Martelossi S, et al. IBD and IBD mimicking enterocolitis in children younger than 2 years of age. Eur J Pediatr 2008; 168: 149-55.

14. Pytrus T, Iwańczak B. Growth retardation in pediatric inflammatory bowel diseases - pathogenesis and treatment. Prz Gastroenterol 2013; 8: 86-92.

15. Mamula P, Telega GW, Markowitz JE, et al. Inflammatory bowel disease in children 5 years of age and younger. Am J Gastroenterol 2002; 97: 2005-10.

16. Levine A, Griffiths A, Markowitz J, et al. Pediatric modification of the Montreal classification for inflammatory bowel disease: the Paris classification. Inflamm Bowel Dis 2011; 17: 1314-21.

17. Dokucu AI, Sarnacki S, Michel JL, et al. Indications and results of surgery in patients with Crohn's disease with onset under 10 years of age: a series of 18 patients. Eur J Pediatr Surg 2002; 12: 180-5.

18. Beque B, Dumant C, Bambou JC, et al. Crohn's disease as an innate immune defect: the critical role of CARD 15 in intestinal antibacterial defense. J Pediatr Gastroentrol Nutr 2004; 39: 345.

19. Gilat T, Hacohen D, Lilos P, Langman MJ. Childhood factors in ulcerative colitis and Crohn's disease. An International Cooperative Study. Scand J Gastroenterol 1987; 22: 1009-24.

20. Wurlemann JI, Lyles CM, Sandler RS. Childhood infections and the risk of inflammatory bowel disease. Dig Dis Sci 1994; 39: 555-60.

Received: 11.01 .2015

Accepted: 26.02 .2015 